MODELING INTERFACIAL THERMAL BOUNDARY CONDUCTANCE OF ENGINEERED INTERFACES

Pamela Norris

UNIVERSITY OF VIRGINIA

09/08/2014

Final Report

DISTRIBUTION A: Distribution approved for public release.

Air Force Research Laboratory

AF Office Of Scientific Research (AFOSR)/ RTB

Arlington, Virginia 22203

Air Force Materiel Command 


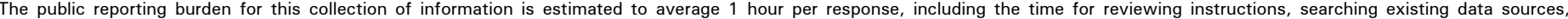

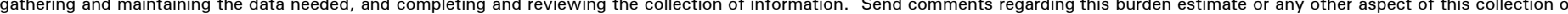

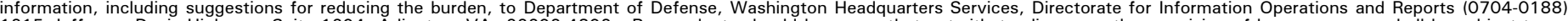

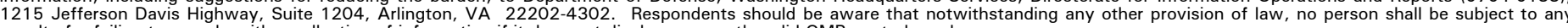
penalty for failing to comply with a collection of information if it does not display a currently valid OMB control number.

PLEASE DO NOT RETURN YOUR FORM TO THE ABOVE ADDRESS.
1. REPORT DATE $(D D-M M-Y Y Y Y)$
2. REPORT TYPE
$08 / 31 / 2014$
FINAL
3. DATES COVERED (From - To)
03/01/09 - 05/31/14

4. TITLE AND SUBTITLE

MODELING INTERFACIAL THERMAL BOUNDARY CONDUCTANCE

OF ENGINEERED INTERFACES

5a. CONTRACT NUMBER

5b. GRANT NUMBER

FA9550-09-1-0245

5c. PROGRAM ELEMENT NUMBER

6. AUTHOR(S)

NORRIS, PAMELA M. 5d. PROJECT NUMBER

5e. TASK NUMBER

5f. WORK UNIT NUMBER

7. PERFORMING ORGANIZATION NAME(S) AND ADDRESS(ES)

RECTOR \& VISITORS OF THE UNIVERSITY OF VIRGINIA

8. PERFORMING ORGANIZATION REPORT NUMBER

UNIVERSITY OF VIRGINIA, OFFICE OF SPONSORED PROGRAMS

P.O. BOX 400195, 1001 NORTH EMMET ST.

CHARLOTTESVILLE, VA 22904-4195

9. SPONSORING/MONITORING AGENCY NAME(S) AND ADDRESS(ES)

AF OFFICE OF SCIENTIFIC RESEARCH

875 NORTH RANDOLPH STREET

ARLINGTON, VA 22203 NUMBER(S)

\section{DISTRIBUTION/AVAILABILITY STATEMENT}

Approved for public release

\section{SUPPLEMENTARY NOTES}

\section{ABSTRACT}

The hypothesis that motivates this grant - that material can be added at an interface to enhance its effective thermal conductancewas first explored using simulations. Our primary tool was the classical molecular dynamics (MD) method. The first part of the simulation work demonstrated that an interfacial film can enhance conductance in simple systems. The second part laid the groundwork to extend those simulations to more complex material systems. To theoretically investigate the phonon transport underlying the conductance trends observed in our simulations, we used various theoretical approaches to understand fundamental phonon transport in interfacial structures: (1) semi-empirical methods such as the DMM, (2) the wavelet transform applied to MD simulations, (3) Green's functions, and (4) the interfering particle model (IPM). Finally, the findings from simulations and theoretical analysis were used to design a series of experimental measurements of hBD at interfaces with varying thicknesses of interfacial films. The findings have been published in the 16 archival journal papers and 3 conference proceeding papers cited here,

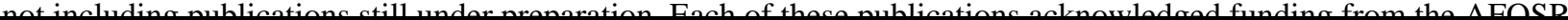
15. SUBJECT TERMS

phonon transport, interfacial thermal transport

16. SECURITY CLASSIFICATION OF:

\begin{tabular}{|l|l|l|}
\hline a. REPORT & b. ABSTRACT & c. THIS PAGE \\
\hline
\end{tabular}

\section{LIMITATION OF ABSTRACT}

UU
18. NUMBER OF PAGES

14 19a. NAME OF RESPONSIBLE PERSON Norris, Pamela M 19b. TELEPHONE NUMBER (Include area code) 4349246295 


\section{AFOSR Final Report}

Project Title:

Award Number:

Program Manager:

Principal Investigator:

Reporting Period:
Modeling Interfacial Thermal Boundary Conductance of Engineered Interfaces

FA9550-09-1-0245

Dr. Jason Marshall / Dr. John Luginsland

RTB-5, Plasma and Electroenergetic Physics

Air Force Office of Scientific Research

875 N. Randolph St.

Arlington, VA 22203

jason.marshall.3@us.af.mil

(703) 696-7721

Dr. Pamela M. Norris

Frederick Tracy Morse Professor

Department of Mechanical and Aerospace Engineering

Associate Dean of Research and Graduate Programs

School of Engineering and Applied Science

University of Virginia

Charlottesville, VA 22904

pamela@virginia.edu

(434) 924-6295

1 March 2009-31 May 2014

\section{Contents}

$\begin{array}{ll}\text { Summary of Accomplishments } & 2\end{array}$

1 Introduction $\quad 3$

2 Simulations: effects of interfacial films on thermal conductance 3

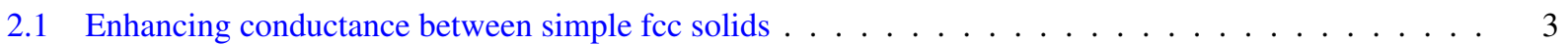

2.2 Transition to realistic interfaces: metals and semiconductors $\ldots \ldots \ldots \ldots \ldots$

3 Theory: understanding conductance via phonon transport theory 5

3.1 Modifying the diffuse mismatch model: single interfaces $\ldots \ldots \ldots \ldots \ldots \ldots$

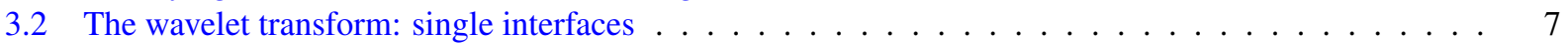

3.3 Green's functions: interfaces with impurities . . . . . . . . . . . . . . . . . . . . . 9

3.4 The interfering particle model: interfaces with intermediate layers $\ldots \ldots \ldots \ldots$

4 Experiments: effects of interfacial films on thermal conductance 11

$\begin{array}{ll}\text { References Acknowledging This Grant } & 13\end{array}$

$\begin{array}{lr}\text { Other References } & 14\end{array}$ 


\section{Summary of Accomplishments}

Simulations: effects of interfacial films on thermal conductance

- Found that inserting an interfacial film at ideal argon-"heavy argon" interfaces can increase $h_{\mathrm{BD}}$ by as much as 23\%. Optimum enhancement occurs at low temperatures ( $10 \%$ of $\left.T_{\text {melt }}\right)$, very thin films ( $<10$ unit cells), and, all else equal, when the film has an atomic mass equal to the average of the abutting materials $[1,2]$.

- Found that small degrees of interfacial mixing can actually further increase $h_{\mathrm{BD}}$. In the best case, enhancement to the baseline $h_{\mathrm{BD}}$ increased from $23 \%$ (sharp interface) to $53 \%$ ("medium" mixing) [2].

- Developed a new interatomic potential, the angular-dependent embedded atom method (A-EAM), to describe multicomponent systems of both metallic and covalent materials [3]. Parameters have been published for the binary systems $\mathrm{Au} / \mathrm{Si}, \mathrm{Au} / \mathrm{Ge}$, and $\mathrm{Al} / \mathrm{Si}$. Parameters for the ternary $\mathrm{Au} / \mathrm{Al} / \mathrm{Si}$ system are to be published.

Theory: understanding conductance via phonon transport theory

- Developed and tested modifications to the diffuse mismatch model (DMM) for predicting $h_{\mathrm{BD}}$ : including optical phonons [4], including full phonon dispersions [5], breaking detailed balance [6], and including anharmonic ("inelastic") phonon processes [7]. Modifications were shown to improve the predictive ability of the DMM.

- Using Green's functions, determined analytical expressions that capture the effects of interfacial bonding and impurities on phonon transmissivity. Used those results to identify cases for optimum transmission [8].

- Applied the wavelet transform to atomistic simulations to reveal phonon transport processes [9]. At interfaces, this approach reveals the effects of mixing and roughness on phonon scattering dynamics.

- Using the interfering particle model, analyzed the effects of film mass and thickness in multilayered structures and found strategies for maximizing and minimizing phonon transmission at interfaces and in superlattices [10].

Experiments: effects of interfacial films on thermal conductance

- Measured the effective conductance $h_{\mathrm{BD}}$ of Pt/Ni/Si samples with varying Ni thickness. Maximum enhancement of $h_{\mathrm{BD}}$ occurred with the thinnest film $(5 \mathrm{~nm}), 145 \%$ higher than in the baseline Pt/Si interface: $\sim 235 \mathrm{vs}$. $95 \mathrm{MW} \mathrm{m}{ }^{-2} \mathrm{~K}^{-1}$. The dramatic enhancement is partially due to bonding, but also partially due to vibrational matching as predicted by simulation. Results to be published.

- Measured Pt/Ni/Ge samples as a counterexample. In the best case $(5 \mathrm{~nm} \mathrm{Ni})$, the effective $h_{\mathrm{BD}}$ is only $50 \%$ higher than that of the baseline $\mathrm{Pt} / \mathrm{Ge}$ interface: $\sim 160$ vs. $105 \mathrm{MW} \mathrm{m}^{-2} \mathrm{~K}^{-1}$. The smaller enhancement is consistent with an increase due to $\mathrm{Ni} / \mathrm{Ge}$ bonding in competition with a decrease due to vibrational mis-match. Results to be published.

The grant also supported several findings related tangentially to the proposed work.

- Investigated thermal transport in systems involving carbon materials. Determined scaling laws for conductivity of carbon nanotube networks [11]. Modified the DMM to predict $h_{\mathrm{BD}}$ at metal-graphite interfaces [12], and showed experimentally that surface treatment can cause $h_{\mathrm{BD}}$ of $\mathrm{Au}$-graphite interfaces to vary by $3 \times[13,14]$.

- Simulated the vibrational contribution to the thermal conductivity of Si near $T_{\text {melt }}$. Vibrational energy transport contributes $\lesssim 4 \%$ of the total conductivity of liquid $\mathrm{Si}$, with electronic transport contributing the rest $[15,16]$.

- Simulated the laser irradiation of a thin $\mathrm{Ag}$ film on $\mathrm{Cu}$ substrate, leading to superfast melting/recrystallization of the subsurface $\mathrm{Ag} / \mathrm{Cu}$ interface. Observed the formation of a novel, lattice-mismatched interfacial microstructure, effectively hardening the interface by laser irradiation [17].

- Simulated the effect of long-range order parameter on the thermal conductivity of crystalline substitutional solid solutions. Found that the long-range order parameter is an effective method for controlling the thermal conductivity in crystalline substitutional solid solutions (e.g., $\mathrm{Si}_{x} \mathrm{Ge}_{1-x}$ alloys) [18].

- Reviewed the research literature regarding methods for tuning heat transport at solid-solid interfaces, superlattices, and heterostructures [19].

These findings have been published in the 16 archival journal papers and 3 conference proceeding papers cited here, not including publications still under preparation. Each of these publications acknowledged funding from the AFOSR. 


\section{Introduction}

The objective of this research was to investigate how the physical aspects of a solid-solid interface affect its thermal conductance $h_{\mathrm{BD}}$, which is expressed in units of $\mathrm{W} \mathrm{m}{ }^{-2} \mathrm{~K}^{-1}$. Researchers also often refer to thermal boundary resistance, which is the inverse quantity. As sketched in Figure 1, $h_{\mathrm{BD}}$ represents an interface's capability to transmit heat flux under a given temperature difference. Typical $h_{\mathrm{BD}}$ at phonon-mediated interfaces (i.e., not metal-metal) is in the range of $10^{1}-10^{2} \mathrm{MW} \mathrm{m}^{-2} \mathrm{~K}^{-1}$.

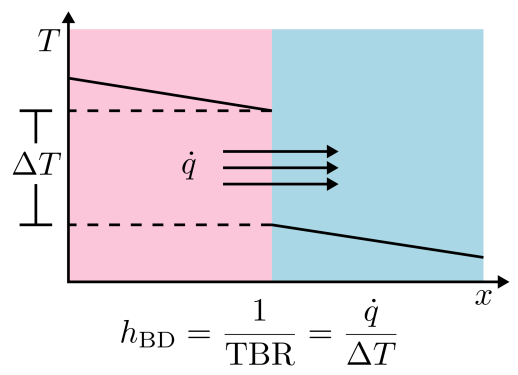

Figure 1: Sketch of a steady-state temperature profile, $T(x)$, in response to a constant heat flux, $\dot{q}$, normal to the interface between two materials. The temperature drop at the interface, $\Delta T$, defines its thermal boundary conductance, $h_{\mathrm{BD}}$. The inverse quantity is known as the thermal boundary resistance (TBR).

Understanding $h_{\mathrm{BD}}$ is crucial in practical applications. For example, the GaN-substrate conductance is a limiting factor in the development of GaN-based high-power, high-frequency devices of interest to the AFOSR [20-23]. Understanding $h_{\mathrm{BD}}$ has also proven to be a very interesting (i.e., difficult) problem in fundamental science, as evidenced by active interest in the topic from the 1940s to the current day [19]. This grant supported complementary computational, theoretical, and experimental investigations of methods for controlling $h_{\mathrm{BD}}$ at an interface by modifying its properties. The methods, results, and conclusions of each investigation are summarized in the following sections.

\section{Simulations: effects of interfacial films on thermal conductance}

The hypothesis that motivates this grant - that material can be added at an interface to enhance its effective thermal conductance-was first explored using simulations. Our primary tool was the classical molecular dynamics (MD) method. The first part of the simulation work demonstrated that an interfacial film can enhance conductance in simple systems. The second part laid the groundwork to extend those simulations to more complex material systems.

\subsection{Enhancing conductance between simple fcc solids}

For details, see Refs. [1] and [2].

\section{Methods}

This work used molecular dynamics (MD) simulations to predict the effects of inserting a thin film at an interface between Ar and "heavy Ar." For each configuration, the effective thermal conductance $h_{\mathrm{BD}}$ was calculated using the non-equilibrium molecular dynamics (NEMD) method. We prescribed a constant heat flux $\left(0.285 \mathrm{GW} \mathrm{m}^{-2}\right)$ through the simulation domain, which leads to the development of a steady-state temperature profile, from which $h_{\mathrm{BD}}$ is readily calculated as sketched in Figure 1. The conductance was calculated in this manner for a range of masses and thicknesses of the film. Once conductance trends were identified, we calculated the local density of states in each material (Ar, heavy Ar, and the film), which provided qualitative explanations of the conductance trends based on phonon transport. 


\section{Results and Conclusions}

A large set of conductance calculations is presented in Figure 2, each normalized relative to the conductance of the baseline Ar-heavy Ar interface with no film. From these data we drew several conclusions:

- With interatomic forces the same throughout, the highest enhancement occurs when the film mass is roughly average. However, the enhancement is not extremely sensitive to mass near the optimum value, which bodes well for materials selection. In real systems with inhomogeneous bonding, we expect that this trend with mass corresponds to a more general trend with vibrational impedance.

- The enhancement decreases monotonically with film thickness-for maximum $h_{\mathrm{BD}}$, make the film not thicker than a few nanometers.

- Mixing at the interface can increase or decrease $h_{\mathrm{BD}}$; there exists an "optimum" degree of mixing, which is shown in the right column of Figure 2. The additional enhancement from mixing is on the same order as the enhancement from the interfacial film itself.

- The enhancement due to the interfacial film is significant at $T=10 \% T_{\text {melt }}$ but vanishes at high temperatures. We attribute this to shorter phonon mean free paths as temperature increases, which changes the transport in the interfacial film from ballistic to diffusive.

\section{Sharp interface}
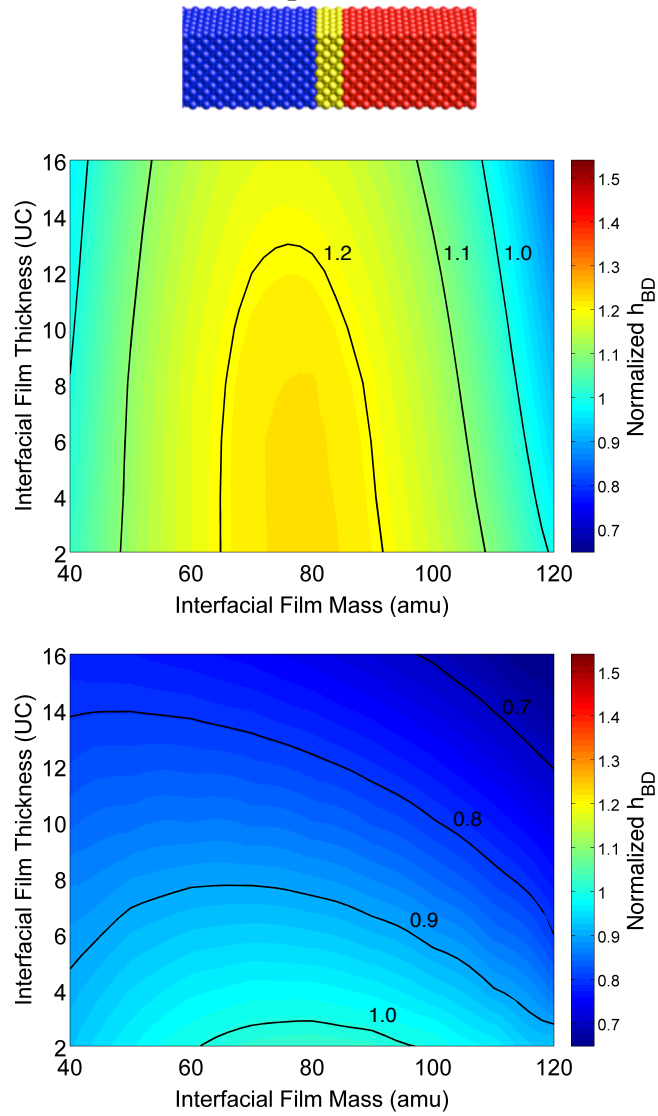

Optimum mixing
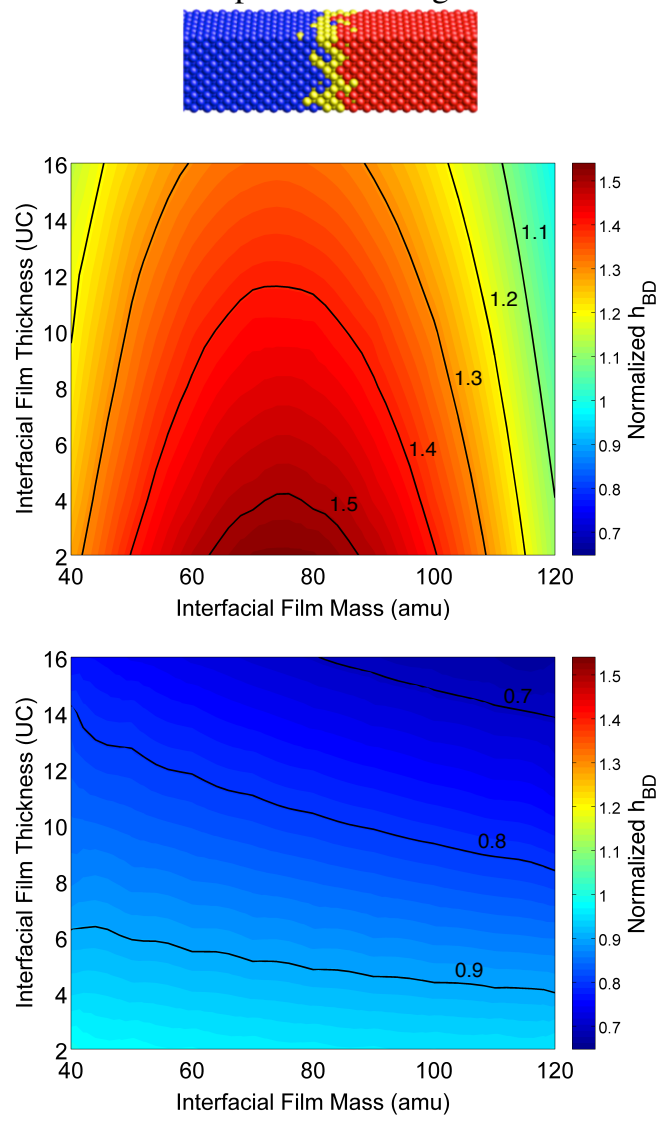

Figure 2: Thermal conductance of various interfaces with interfacial films, normalized by the conductance of the baseline Ar-heavy Ar interface. The data span four independent variables: film mass, film thickness, degree of interfacial mixing, and overall system temperature. 


\subsection{Transition to realistic interfaces: metals and semiconductors}

For details about the formulation of the angular-dependent embedded atom method (A-EAM), and for parameters describing the binary $\mathrm{Au}-\mathrm{Si}$ and $\mathrm{Au}-\mathrm{Ge}$ systems, see Ref. [3]. For a parameterization of the Al-Si system, also see Ref. [24], which was not supported by this grant. We also present a new preliminary parameter set for the Al-Si system, not yet published, which can be used with the Au-Si parameter set to describe the ternary Au-Al-Si system.

\section{Methods}

The general form of the A-EAM was developed by combining the existing Stillinger-Weber (SW) potential for tetrahedrally coordinated materials [25] and the embedded-atom method (EAM) potential for metals [26, 27]. The resulting potential functions are models for interatomic forces among both covalent and metallic species; for example, in the binary $\mathrm{Au}-\mathrm{Si}$ system. (A potential must be available for each pure component, either in the SW form for a covalent material or in the EAM form for a metal.) The new A-EAM potential requires the appropriate selection of values for 14 parameters for each binary system.

For each binary system, those parameters must be chosen to reproduce reference data of interest to the simulator. In our case, we are interested in reproducing interatomic interactions in the solid state. Unfortunately, little experimental information is available for systems like $\mathrm{Au}-\mathrm{Si}$ that are immiscible in the solid state, so we performed sets of calculations using density functional theory (DFT) to supplement experimental data. The calculations in Ref. [3] were performed using the Vienna ab initio simulation package (VASP), and the calculations for the ternary Au-Al-Si system (presented below, not yet published) were performed using Quantum Espresso. The latter calculations were converged within $1 \times 10^{-4}$ Ryd with respect to wave function cutoff energy, energy density cutoff, and $k$-point sampling.

The A-EAM parameters were then chosen to best reproduce the lattice constants $a$, cohesive energies $E$, and bulk moduli $B$ of crystalline structures as well as formation energies of vacancies $E_{\text {vac }}$ and substitutional impurities $E_{\text {sub. }}$. In practice, three of the parameters are held constant, as described in Ref. [3], so each binary system has 11 free fitting parameters.

\section{Results and Conclusions}

- A new interatomic potential, the A-EAM, was developed for multicomponent systems of mixed metallic and covalent species.

- Using a series of DFT calculations as fitting targets, parameters have been determined for interactions between $\mathrm{Au}-\mathrm{Si}, \mathrm{Au}-\mathrm{Ge}$, and $\mathrm{Al}-\mathrm{Si}$ atoms, as summarized in Table 1. Those potential parameters give rise to simulated material properties listed in Table 2, where they are compared with reference values (either experimental or DFT). The properties of three additional structures, omitted here for brevity, were also used for the fitting process: $\mathrm{AuSi}, \mathrm{AlSi}$, and $\mathrm{Au}_{4} \mathrm{Al}$. In addition to reproducing behavior in the solid state, the potentials are also shown to accurately reproduce enthalpies of mixing in the liquid state.

- The Al-Si parameters reported here are compatible with the Au-Si parameters and can be used simultaneously to describe the ternary Au-Al-Si system by applying the alloying rules described by Zhou et al. [28]. This is not true of the Al-Si parameters presented, for example, in Ref. [24].

\section{Theory: understanding conductance via phonon transport theory}

To investigate the phonon transport underlying the conductance trends observed in Section 2, we used various theoretical approaches to understand fundamental phonon transport in interfacial structures: (1) semi-empirical methods such as the DMM, (2) the wavelet transform applied to MD simulations, (3) Green's functions, and (4) the interfering particle model (IPM).

\subsection{Modifying the diffuse mismatch model: single interfaces}

Regarding the use of full phonon dispersions instead of the Debye approximation, see Ref. [5]. For details about modifying the diffuse mismatch model (DMM) to include the effects of optical phonons, see Ref. [4]. Regarding the 
Table 1: A-EAM Parameters and Fitted Values for Selected Binary Systems

\begin{tabular}{lccc} 
Parameter & $\mathrm{Au}-\mathrm{Si}$ & $\mathrm{Au}-\mathrm{Ge}$ & $\mathrm{Al}-\mathrm{Si}$ \\
\hline$A_{C}(\mathrm{eV})$ & 5.189 & 4.769 & 3.414 \\
$B_{C}(\mathrm{eV})$ & 9.8129 & 8.779 & 5.751 \\
$\alpha_{C}$ & -0.555 & -0.555 & -0.517 \\
$\beta_{C}$ & -0.93 & -0.93 & -0.637 \\
$\sigma_{C}(\AA)$ & 1.81 & 1.884 & 2.313 \\
$P_{C}$ & 4 & 4 & 4 \\
$Q_{C}$ & 0 & 0 & 0 \\
$f_{e}^{\text {metal }}$ & 4.8 & 4.2 & 1.120 \\
$f_{e}^{\text {covalent }}$ & 16 & 16 & 16 \\
$\gamma_{C}^{\text {metal }}$ & 3.56 & 3.56 & 3.499 \\
$\gamma_{C}^{\text {covalent }}$ & -1.1 & -1.1 & -3.130 \\
$R_{e}^{\text {metal }}$ & 2.47 & 2.57 & 3.229 \\
$R_{S}(\AA)$ & 2.97 & 3.07 & 3.073 \\
$R_{M}(\AA)$ & 4.7837 & 4.98 & 4.390
\end{tabular}

Table 2: Evaluation of Selected Material Properties Predicted by the A-EAM

\begin{tabular}{|c|c|c|c|c|c|}
\hline Binary System & Structure & Property & A-EAM & Reference & \\
\hline \multirow[t]{6}{*}{$\mathrm{Au}-\mathrm{Si}$} & \multirow[t]{6}{*}{$\mathrm{Au}_{3} \mathrm{Si}$} & $a(\AA)$ & 4.193 & $4.145^{\ddagger}$ & \multirow[t]{12}{*}{ [3] } \\
\hline & & & & $4.087^{\dagger}$ & \\
\hline & & $E(\mathrm{eV})$ & -4.035 & $-3.149^{\ddagger}$ & \\
\hline & & & & $-4.108^{\dagger}$ & \\
\hline & & $B$ (Mbar) & 1.591 & $1.203^{\ddagger}$ & \\
\hline & & & & $1.888^{\dagger}$ & \\
\hline \multirow[t]{6}{*}{$\mathrm{Al}-\mathrm{Si}$} & \multirow[t]{6}{*}{$\mathrm{Al}_{3} \mathrm{Si}$} & $a$ & 3.848 & $4.145^{\ddagger}$ & \\
\hline & & & & $4.087^{\dagger}$ & \\
\hline & & $E$ & -4.252 & $-3.149^{\ddagger}$ & \\
\hline & & & & $-4.108^{\dagger}$ & \\
\hline & & $B$ & 1.172 & $1.203^{\ddagger}$ & \\
\hline & & & & $1.888^{\dagger}$ & \\
\hline \multirow[t]{3}{*}{$\mathrm{Au}-\mathrm{Al}$} & \multirow[t]{3}{*}{$\mathrm{AuAl}_{2}$} & $a$ & 6.102 & $6.070^{*}$ & [29] \\
\hline & & E & -3.416 & $-3.149^{*}$ & [30] \\
\hline & & $B$ & 0.794 & $1.030^{*}$ & [29] \\
\hline${ }^{*}$ Experimental & & & & & \\
\hline$\ddagger$ DFT calcul & ing ( & & & & \\
\hline
\end{tabular}


relaxation of the assumption of detailed balance, see Ref. [6]. Regarding the effects of inelastic phonon transmission, see Ref. [7].

\section{Methods}

The DMM predicts the conductance at an interface based on the carrier energy $\hbar \omega$, density of states $D$, occupation $f_{0}$, group velocity $v$, and transmission probability $\tau$ of each phonon frequency [31]:

$$
h_{\mathrm{BD}}=\frac{1}{4} \sum_{j}^{3} \int_{0}^{\omega_{D, j}} \tau_{j}(\omega) \hbar \omega v_{1, j}(\omega) D_{j}(\omega) \frac{\partial f_{0}(\omega)}{\partial T} d \omega .
$$

The problem then becomes how to calculate $\tau$, since the other quantities are based only on bulk material properties.

\section{Results and Conclusions}

- Using full phonon dispersions rather than the usual Debye approximation can lead to such and such improvement in predictions of $h_{\mathrm{BD}}$ in some systems.

- Contrary to common assumption, the transmission of optical phonons contributes a large fraction toward the total conductance at many interfaces (Figure 3, left). The optical phonon contribution correlates with the number of optical branches in the abutting materials; i.e., with the complexity of their unit cells.

- The assumption of strict detailed balance when calculating the transmission probability $\tau$ can lead to poor predictions of $h_{\mathrm{BD}}$. Predictions can be significantly improved by assuming "quasi-elastic" scattering (Figure 3, right).

- We developed an anharmonic inelastic model (AIM) which modifies the DMM to account inelastic 4-phonon, 5-phonon, etc. processes in a detailed manner. Agreement with experimental $h_{\mathrm{BD}}(T)$ data is significantly improved.
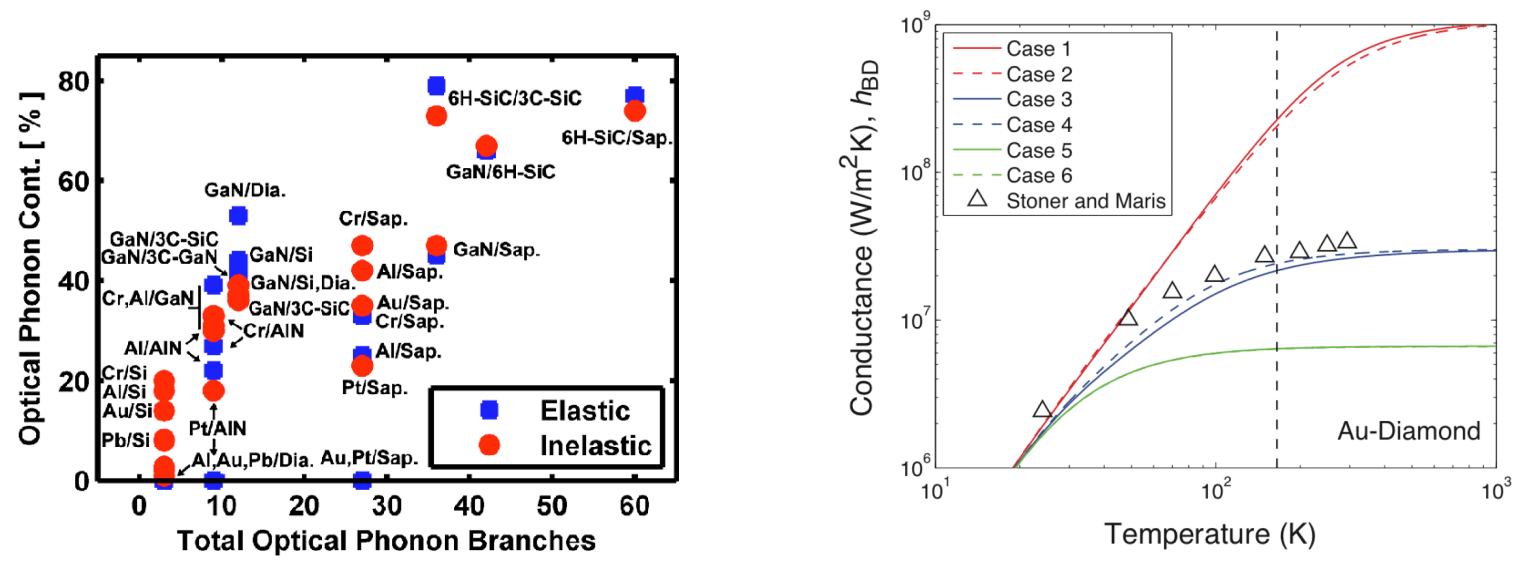

Figure 3: Findings regarding the phonon transport underlying $h_{\mathrm{BD}}$, based on the DMM. Left: the fraction of total $h_{\mathrm{BD}}$ contributed by the transmission of optical phonons at various interfaces, sorted by the total number of optical branches in the constituent materials. Right: comparison of experimental $h_{\mathrm{BD}}(T)$ data with DMM predictions using different assumptions for calculating $\tau(\omega)$.

\subsection{The wavelet transform: single interfaces}

For details, see Ref. [9]. 


\section{Methods}

The wavelet transform is akin to the Fourier transform, except that the basis function used for the transform is localized in both position and wavenumber. Thus, the wavelet transform can reveal the phonon dynamics that are implicitly present in a molecular dynamics simulation. The wave-packet method was used to study anharmonic phonon decay and one-dimensional NEMD was used to study phonon dynamics during transport at an interface.

\section{Results and Conclusions}

The wavelet transform makes no assumptions regarding the underlying physics of the simulation, and thus accommodates systems where anharmonicity is important. Figure 4 shows the application of the wavelet transform to analyze phonon dynamics in two scenarios:

1. Left: the anharmonic decay of a large-amplitude phonon. The wavelet transform reveals how the energy distribution among wavenumbers changes in time and space. Energy first transfers into the $2 k_{p}$ range, representing a 3-phonon normal scattering process. More chaotic interactions then occur, distorting the initial wave-packet's shape and introducing energy at greater wavenumbers. The wave-packet method is quite artificial and meant to isolate a particular physical process. But the wavelet transform can also accommodate a finite-temperature system, which can be thought of as an ensemble of wave-packets.

2. Right: the distribution of energy among vibrational modes near an interface in thermal non-equilibrium. The interatomic forces in the system are purely harmonic. The wavelet transform indicates that the cause of thermal boundary resistance is the population of phonons in side 1 that lie above the cutoff frequency of side 2, corresponding to $q \approx 0.4 q_{\max }$. Instead of transmitting, these phonons accumulate in side 1 . The wavelet transform thus illustrates the underlying physics of the boundary resistance, which this grant has addressed.
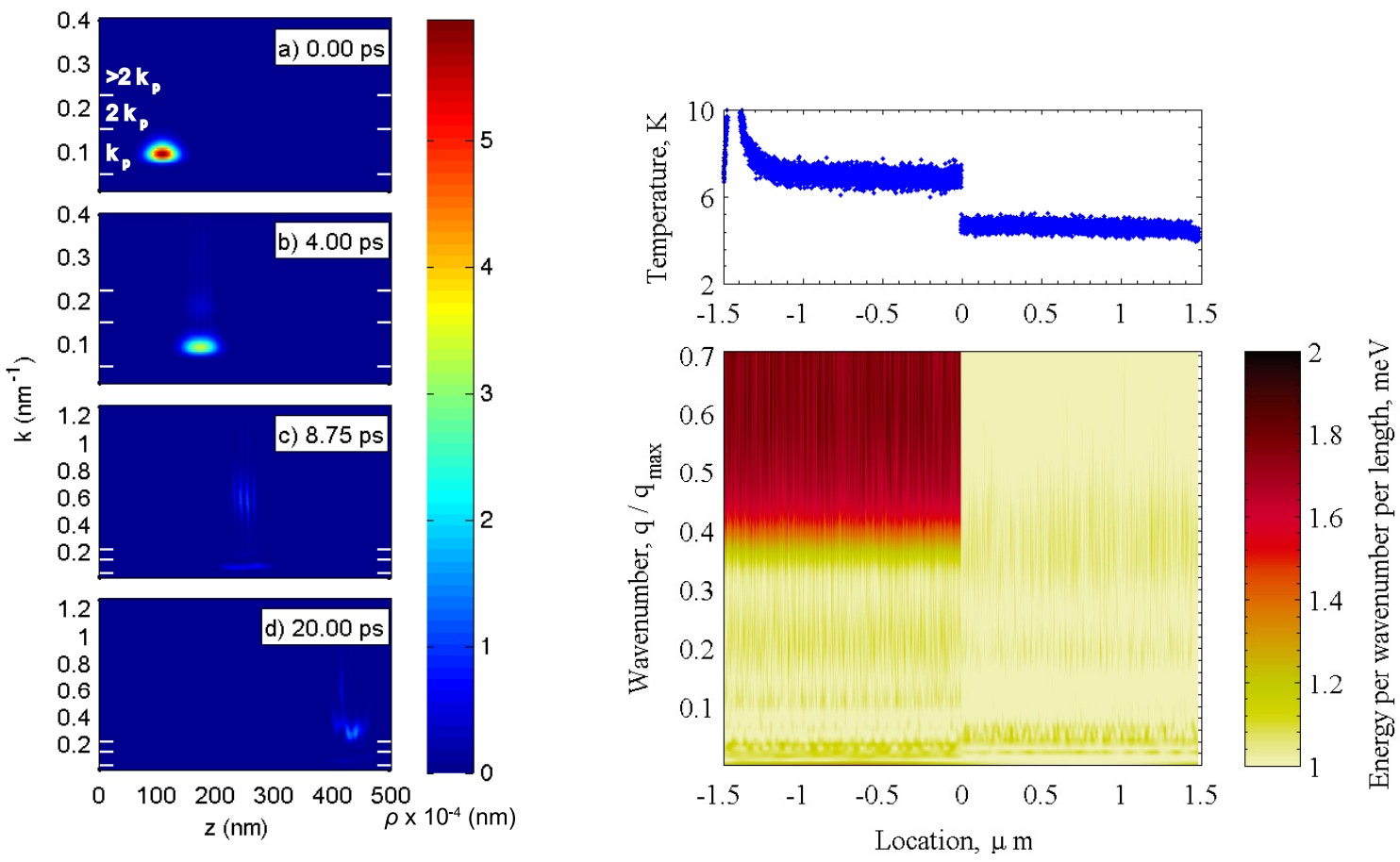

Figure 4: Applications of the wavelet transform to discern phonon dynamics during MD simulations. Left: the anharmonic decay of vibrational energy in a wave packet into modes of different wavenumbers. Right: the spatial variation of the vibrational energy spectrum during a NEMD simulation at an interface. 
In conclusion:

- The wavelet transform gives spatially localized spectral information from molecular dynamics simulations.

- It is a flexible tool that can be used to investigate individual scattering events (phonon-phonon, phonon-interface), finite-temperature phonon non-equilibrium, and even carrier dynamics in non-crystalline materials.

\subsection{Green's functions: interfaces with impurities}

For details, see Ref. [8].

\section{Methods}

The formulation for Green's functions, as applied here, is based on a system of classical harmonic Newtonian equations which describes the forces acting on each atom in a one-dimensional system. The model system is comprised of three parts: the source, the sink, and a center region (the "channel"). In general, the source and sink are two different semiinfinite materials, having different masses and spring constants, and the channel is some finite sequence of masses and springs.

Using the Green's function method and the Caroli formula, we solve the system of Newton's equations to produce analytical expressions for the energy transmission ratio as a function of vibrational frequency. Those expressions were used to study the effects of tuning "impurity" masses and springs on phonon transmission.

\section{Results and Conclusions}

- At an interface between materials $1 \& 2$ with an impurity mass $m_{\text {int }}$, the energy transmission of all phonon frequencies is maximized when $m_{\text {int }}=\left(m_{1}+m_{2}\right) / 2$, regardless of $k_{1}$ and $k_{2}$ (Figure 5, left).

- In that case, the analytical expression for the transmission takes precisely the form of the acoustic mismatch model (AMM), which predicts transmission based on the acoustic impedance of each material. However, this Green's function approach leads to a different expression for impedance, leading to a transmission expression that is true for all phonon frequencies, unlike the AMM.

- With an impurity spring $k_{\text {int }}$, the energy transmission of all phonon frequencies is maximized when $k_{\text {int }}=$ $2 k_{1} k_{2} /\left(k_{1}+k_{2}\right)$ (Figure 5, right).

- In that case, the analytical expression for the transmission does not take the form of the AMM. Rather, the expression includes an additional resonance term that depends on the properties of the source and sink.
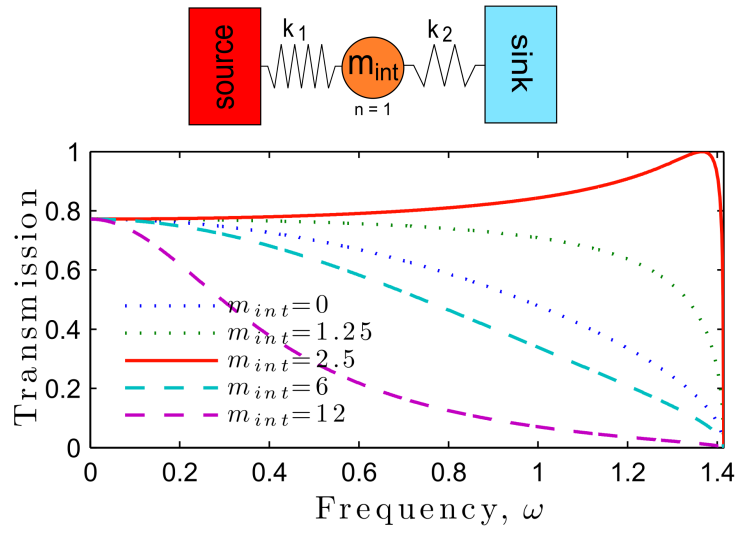
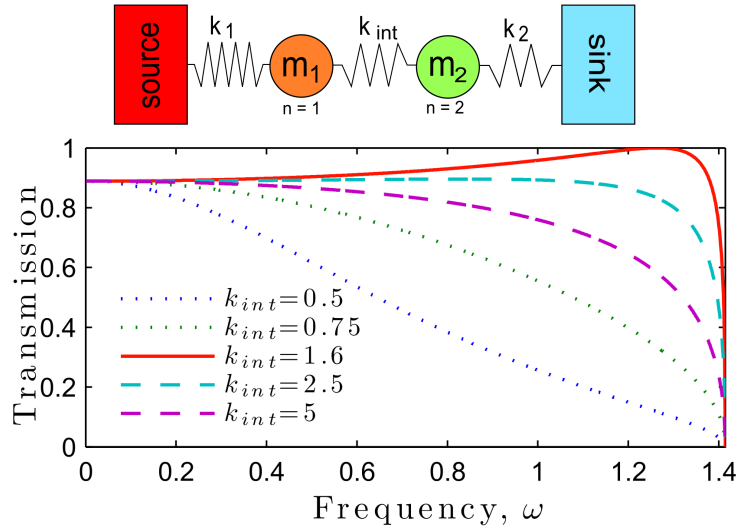

Figure 5: Phonon transmission spectra, with curves corresponding to different impurity masses, $m_{\text {int }}$ (left plot) or different bonding strengths, $k_{\text {int }}$ (right plot). 


\subsection{The interfering particle model: interfaces with intermediate layers}

For details, see Ref. [10].

\section{Methods}

The interfering particle model (IPM) [32] was used to simulate the propagation of vibrational energy through interfacial structures with $N$ intermediate layers. In the original implementation of the IPM, Schelling and Phillpot obtained the phonon transmissivity at each of the $N-1$ interfaces by using MD wave packet simulations, which were by far the dominant computational expense. In the current work, the phonon transmissivities were obtained instead from an algebraic expression that accurately reproduces the MD results but with negligible computational expense. This permitted a large parameter sweep of layer masses and phonon frequencies in structures with $N=1$ (93 simulations), $N=2$ (961 simulations), and $N=4$ (923 521 simulations). Each simulation provides the net energy transmission ratio for a particular phonon frequency through a particular multilayer structure. The results were searched to identify effective strategies for maximizing and minimizing phonon transport through mulitayer structures.

\section{Results and Conclusions}

Selected transmissivity results are shown in Figure 6 in structures with $N=1$ intermediate layer (left) and structures with $N=4$ layers (right). The transmissivity in the $N=4$ structure is calculated for the low-frequency case, $\omega=4.59 \operatorname{Trad~s}^{-1}$. From these data we drew several conclusions.

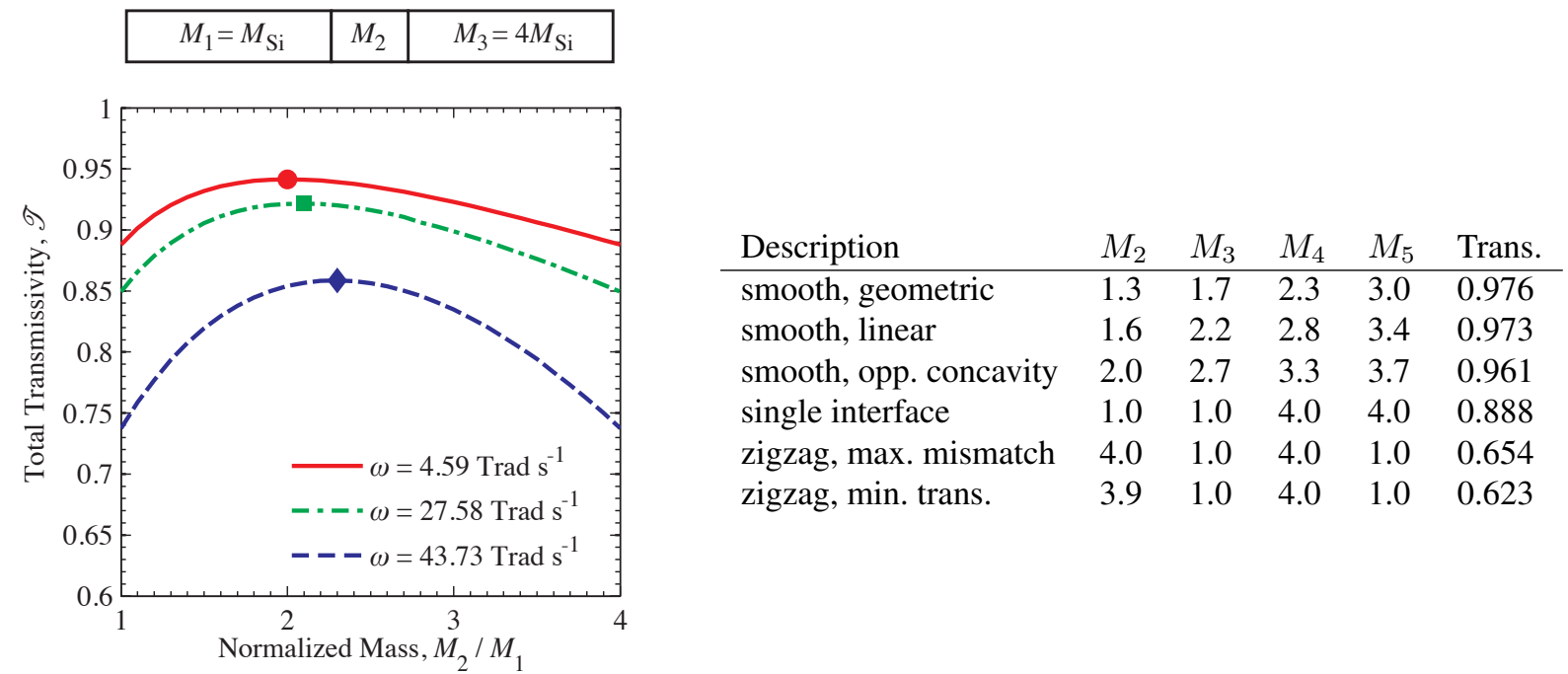

Figure 6: Selected phonon transmissivity data predicted using the IPM in structures with $N=1$ intermediate layer (left plot) and in structures with $N=4$ layers (right table). In both cases, the left lead has normalized mass $M_{1}=1$, and the right lead has $M_{N+2}=4$.

- In the structure with $N=1$ layer, choosing the layer mass as the geometric mean maximizes the energy transmission of low-frequency phonons. For higher phonon frequencies, the optimum choice of mass shifts upward toward the arithmetic mean. Therefore, the choice of layer mass that maximizes the effective $h_{\mathrm{BD}}$ is likely in between.

- In the structure with $N=4$ layers, transmission is maximized when the layer masses change monotonically (labeled "smooth"). The optimum transmissivity occurs when the sequence of masses is geometric, providing a significant enhancement compared to the single-interface transmission. However, this enhancement is not particularly sensitive to the exact sequence as long as the transition is "smooth." 
- The transmission is minimized when the layer masses alternately increase and decrease ("zigzag"), as in a superlattice. However, the minimum transmissivity does not necessarily occur in the sequence with the greatest impedance mismatch ("max. mismatch"), since some constructive interference can occur. Instead, the minimum transmissivity occurs in an aperiodic structure which sacrifices some degree of impedance mismatch to disrupt constructive interference.

\section{Experiments: effects of interfacial films on thermal conductance}

Finally, the findings from simulations and theoretical analysis were used to design a series of experimental measurements of $h_{\mathrm{BD}}$ at interfaces with varying thicknesses of interfacial films.

\section{Methods}

The effective thermal boundary conductance of each metal-substrate system was measured using time domain thermoreflectance (TDTR), an optical pump-probe technique that is well established for measuring thermophysical properties of systems with nanoscale features [33]. Two series of samples were fabricated at the Army Research Laboratory in collaboration with the US Naval Academy: (1) a series of Pt-Ni-Si samples, in which the thickness of the Ni layer was varied from 5 to $100 \mathrm{~nm}$, and (2) a corresponding series of Pt-Ni-Ge samples. The choice of materials was based in part on the ratios of the Debye temperatures $\theta_{D}$ of the intermediate layer and the substrate $[2,34]$. The thermal decay of each sample, measured by TDTR, was compared to a multilayer solution of the heat diffusion equation to extract the conductance $h_{\mathrm{BD}}$ between $\mathrm{Ni}$ and $\mathrm{Si}(\mathrm{Ge})$ [35]. Using a series thermal resistor analysis, the total Pt-tosubstrate conductance is calculated. That total conductance is compared with the baseline measurements of $h_{\mathrm{BD}}$ at $\mathrm{Pt}-\mathrm{Si}$ and $\mathrm{Pt}-\mathrm{Ge}$ interfaces without an intermediate layer.

\section{Results and Conclusions}

Figure 7 shows the resulting $h_{\mathrm{BD}}$ measurements as a function of intermediate layer thickness for each of the two material systems. From these results we drew several conclusions:

- The Pt-Ni-Si system shows drastically enhanced conductance compared to the Pt-Si interface. The enhancement is best for the $5 \mathrm{~nm}$ Ni layer and decreases with increasing thickness, as predicted by the MD simulations summarized in Section 2.

- Some of the enhanced $h_{\mathrm{BD}}$ can be attributed to increased bonding between Ni-Si (vs. Pt-Si). The remainder of the enhancement is due to the increased vibrational match: the Ni layer has a Debye temperature in between the $\mathrm{Pt}$ and Si leads.

- In the Pt-Ni-Ge system, $h_{\mathrm{BD}}$ is enhanced slightly when the Ni layer is very thin. However, as thickness increases, quickly falls below the baseline Pt-Ge conductance. We attribute this behavior to enhanced $\mathrm{Ni}-\mathrm{Ge}$ bonding in competition with a vibrational mismatch: the intermediate Ni layer has a higher Debye temperature than both of the Pt and Ge leads.

- The large enhancement observed in the Pt-Ni-Si system and the small enhancement in the Pt-Ni-Ge system provide strong evidence that $h_{\mathrm{BD}}$ can be enhanced at mismatched interfaces by inserting additional material of intermediate properties.

The experimental work summarized here is under preparation to be published. 

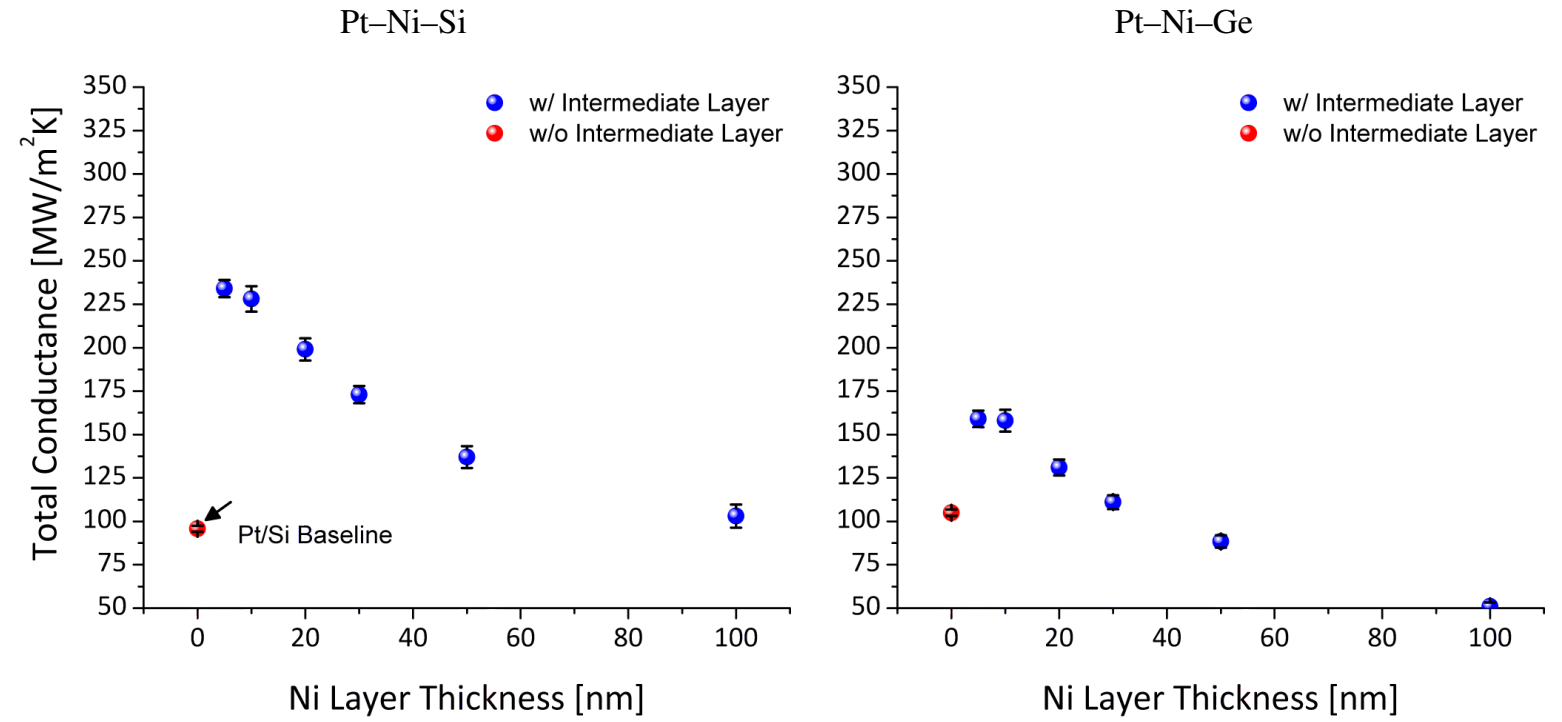

Figure 7: Effective conductance of Pt-Ni-semiconductor samples with varying thickness of the Ni layer. The Ni layer is expected to "bridge" the vibrational mismatch betwen Pt and Si (left), but not between Pt and Ge (right). 


\section{References Acknowledging This Grant}

${ }^{1}$ T. S. English, J. C. Duda, D. A. Jordan, P. M. Norris, and L. V. Zhigilei, "The effect of interstitial layers on thermal boundary conductance between Lennard-Jones crystals", in Proceedings of 14th International Heat Transfer Conference, Vol. 6 (ASME, 2010), pp. 443-448.

${ }^{2}$ T. S. English, J. C. Duda, J. L. Smoyer, D. A. Jordan, P. M. Norris, and L. V. Zhigilei, "Enhancing and tuning phonon transport at vibrationally mismatched solid-solid interfaces", Physical Review B 85, 035438 (2012).

${ }^{3}$ A. M. Dongare, M. Neurock, and L. V. Zhigilei, "Angular-dependent embedded atom method potential for atomistic simulations of metal-covalent systems", Physical Review B 80, 184106 (2009).

${ }^{4}$ T. Beechem, J. C. Duda, P. E. Hopkins, and P. M. Norris, "Contribution of optical phonons to thermal boundary conductance”, Applied Physics Letters 97, 061907 (2010).

${ }^{5}$ J. C. Duda, T. E. Beechem, J. L. Smoyer, P. M. Norris, and P. E. Hopkins, "Role of dispersion on phononic thermal boundary conductance", Journal of Applied Physics 108, 073515 (2010).

${ }^{6}$ J. C. Duda, P. E. Hopkins, J. L. Smoyer, M. L. Bauer, T. S. English, C. B. Saltonstall, and P. M. Norris, "On the assumption of detailed balance in prediction of diffusive transmission probability during interfacial transport", Nanoscale and Microscale Thermophysical Engineering 14, 21-33 (2010).

${ }^{7}$ P. E. Hopkins, J. C. Duda, and P. M. Norris, "Anharmonic phonon interactions at interfaces and contributions to thermal boundary conductance", Journal of Heat Transfer 133, 062401 (2011).

${ }^{8}$ C. B. Saltonstall, C. A. Polanco, J. C. Duda, A. W. Ghosh, P. M. Norris, and P. E. Hopkins, "Effect of interface adhesion and impurity mass on phonon transport at atomic junctions", Journal of Applied Physics 113, 013516 (2013).

${ }^{9}$ C. H. Baker, D. A. Jordan, and P. M. Norris, "Application of the wavelet transform to nanoscale thermal transport", Physical Review B 86, 104306 (2012).

${ }^{10}$ N. Q. Le, J. C. Duda, T. S. English, P. E. Hopkins, T. E. Beechem, and P. M. Norris, "Strategies for tuning phonon transport in multilayered structures using a mismatch-based particle model", Journal of Applied Physics 111, 084310 (2012).

${ }^{11}$ A. N. Volkov and L. V. Zhigilei, "Scaling laws and mesoscopic modeling of thermal conductivity in carbon nanotube materials", Physical Review Letters 104, 215902 (2010).

${ }^{12}$ J. C. Duda, P. E. Hopkins, T. E. Beechem, J. L. Smoyer, and P. M. Norris, "Inelastic phonon interactions at solidgraphite interfaces", Superlattices and Microstructures 47, 550-555 (2010).

${ }^{13}$ P. M. Norris, J. L. Smoyer, J. C. Duda, and P. E. Hopkins, "Prediction and measurement of thermal transport across interfaces between isotropic solids and graphitic materials", in Proceedings of ASME FEDSM2010-ICNMM2010 (2010), p. 30171.

${ }^{14}$ P. M. Norris, J. L. Smoyer, J. C. Duda, and P. E. Hopkins, "Prediction and measurement of thermal transport across interfaces between isotropic solids and graphitic materials", Journal of Heat Transfer 134, 020910 (2012).

${ }^{15}$ C. H. Baker, C. Wu, R. N. Salaway, L. V. Zhigilei, and P. M. Norris, "Vibrational contribution to thermal conductivity of silicon near solid-liquid transition", in Proceedings of ASME International Mechanical Engineering Congress and Exposition, Vol. 10 (ASME, 2011), pp. 351-355.

${ }^{16}$ C. H. Baker, C. Wu, R. N. Salaway, L. V. Zhigilei, and P. M. Norris, "Resolving the vibrational and electronic contributions to thermal conductivity of silicon near the solid-liquid transition: molecular dynamics study", International Journal of Transport Phenomena 13, 143-150 (2013).

${ }^{17}$ C. Wu, D. A. Thomas, Z. Lin, and L. V. Zhigilei, "Runaway lattice-mismatched interface in an atomistic simulation of femtosecond laser irradiation of Ag film-Cu substrate system", Applied Physics A 104, 781-792 (2011).

${ }^{18}$ J. C. Duda, T. S. English, D. A. Jordan, P. M. Norris, and W. A. Soffa, "Controlling thermal conductivity of alloys via atomic ordering", Journal of Heat Transfer 134, 014501 (2012).

${ }^{19}$ P. M. Norris, N. Q. Le, and C. H. Baker, “Tuning phonon transport: from interfaces to nanostructures”, Journal of Heat Transfer 135, 061604 (2013). 


\section{Other References}

${ }^{20}$ R. T. Kemerley, H. B. Wallace, and M. N. Yoder, "Impact of wide bandgap microwave devices on DoD systems", Proceedings of the IEEE 90, 1059-1064 (2002).

${ }^{21} \mathrm{~V}$. O. Turin and A. A. Balandin, "Performance degradation of GaN field-effect transistors due to thermal boundary resistance at GaN/substrate interface", Electronics Letters 40, 81-83 (2004).

${ }^{22}$ A. Sarua, H. Ji, K. P. Hilton, D. J. Wallis, M. J. Uren, T. Martin, and M. Kuball, "Thermal boundary resistance between GaN and substrate in AlGaN/GaN electronic devices", IEEE Transactions on Elecron Devices 54, 31523158 (2007).

${ }^{23}$ J. Kuzmík, S. Bychikhin, D. Pogany, C. Gaquière, E. Pichonat, and E. Morvan, "Investigation of the thermal boundary resistance at the III-nitride/substrate interface using optical methods", Journal of Applied Physics 101, 054508 (2007).

${ }^{24}$ A. M. Dongare, B. LaMattina, D. L. Irving, A. M. Rajendran, M. A. Zikry, and D. W. Brenner, "An angulardependent embedded atom method (A-EAM) interatomic potential to model thermodynamic and mechanical behavior of Al/Si composite materials", Modelling and Simulation in Materials Science and Engineering 20, 035007 (2012).

${ }^{25} \mathrm{~F}$. H. Stillinger and T. A. Weber, "Computer simulation of local order in condensed phases of silicon", Physical Review B 31, 5262-5271 (1985).

${ }^{26}$ M. S. Daw and M. I. Baskes, "Semiempirical, quantum mechanical calculation of hydrogen embrittlement in metals", Physical Review Letters 50, 1285-1288 (1983).

${ }^{27}$ M. S. Daw and M. I. Baskes, "Embedded-atom method: derivation and application to impurities, surfaces, and other defects in metals", Physical Review B 29, 6443-6453 (1984).

${ }^{28}$ X. W. Zhou, H. N. G. Wadley, R. A. Johnson, D. J. Larson, N. Tabat, A. Cerezo, A. K. Petford-Long, G. D. W. Smith, P. H. Clifton, R. L. Martens, and T. F. Kelly, "Atomic scale structure of sputtered metal multilayers", Acta Materialia 49, 4005-4015 (2001).

${ }^{29}$ L.-S. Hsu, Y.-K. Wang, Y.-L. Tai, and J.-F. Lee, "Experimental and theoretical study of the electronic structure of $\mathrm{AuAl}_{2}, \mathrm{AuGa}_{2}$, and AuIn 2 ", Physical Review B 72, 115115 (2005).

${ }^{30}$ R. Pretorius, A. M. Vredenberg, F. W. Saris, and R. de Reus, "Prediction of phase formation sequence and phase stability in binary metal-aluminum thin-film systems using the effective heat of formation rule", Journal of Applied Physics 70, 3636-3646 (1991).

${ }^{31}$ E. T. Swartz and R. O. Pohl, "Thermal boundary resistance”, Reviews of Modern Physics 61, 605-668 (1989).

${ }^{32} \mathrm{P}$. K. Schelling and S. R. Phillpot, "Multiscale simulation of phonon transport in superlattices", Journal of Applied Physics 93, 5377 (2003).

${ }^{33}$ P. M. Norris, A. P. Caffrey, R. J. Stevens, J. M. Klopf, J. T. McLeskey Jr., and A. N. Smith, "Femtosecond pumpprobe nondestructive examination of materials (invited)", Review of Scientific Instruments 74, 400-406 (2003).

${ }^{34}$ Z. Liang and H.-L. Tsai, "Effect of interlayer between semiconductors on interfacial thermal transport", in Proceedings of the ASME 2012 3rd Micro/Nanoscale Heat \& Mass Transfer International Conference (2012).

${ }^{35} \mathrm{~A}$. Feldman, "Algorithm for solutions of the thermal diffusion equation in a stratified medium with a modulated heating source", High Temperatures - High Pressures 31, 293-298 (1999). 